\title{
OLUtool als neues Einheitsmodell der Vertrauensärzte
}

Robert Fries, Yvonne Hummel, Beat Kipfer, Ursula Schafroth, Beat Seiler und Jürg Zollikofer

alle Dr. med., Vertrauensarzt SGV

OLUtool, ein Modell zur Nutzenbestimmung für Arzneimittel im Art. 71 a-d KVV, ist die Antwort auf die Kritik, dass die Beurteilung im OLU-Bereich nicht einheitlich sei. OLUtool ermöglicht eine schnelle und transparente Nutzenbeurteilung, wie dies im klinischen Alltag verlangt wird. Das Modell basiert auf international anerkannten Kriterien, welche klar definiert sind, und steht im Einklang mit der schweizerischen Rechtsprechung. Präsentationen bei Ärzteschaft und Pharmaindustrie stossen auf positive Akzeptanz. Die Trennung zwischen Studienrating und Einzelfall ermöglicht neue Perspektiven für die Zukunft.

Im März 2011 wurden die Kassenpflicht für Arzneimittel ausserhalb der Spezialitätenliste (SL) und deren Limitationen im Art. 71 der KVV neu geregelt. Damit soll den aktuellen wissenschaftlichen Erkenntnissen und der Dynamik der Pharmaforschung begegnet und ein früher Zugang zu hochwirksamen medikamentösen Behandlungen gesichert werden.

Der Vertrauensarzt wird beauftragt, die dringende Notwendigkeit des Arzneimittels und dessen Nutzen im Einzelfall zu bestimmen. Der Krankenversicherer hat nach Feststellung eines erwartbaren grossen Nutzens im Einzelfall eine angemessene Vergütung zu entrichten. Von Patientenorganisationen und Ärzteschaft wird kritisiert, dass die Einheitlichkeit der Beurteilung und damit die Zugangsgerechtigkeit ungenügend seien. Die Kritik ist gerechtfertigt und hat mehrere Ursachen, insbesondere weil Art. 71 a-d KVV sehr offen formuliert ist

\section{Résumé}

OLUtool est un modèle d'évaluation du bénéfice des médicaments dans le cadre de l'art. 71 a-d OAMal, créé en réponse à la critique du manque d'uniformité de l'évaluation dans le domaine OLU. OLUtool permet une détermination rapide et transparente du bénéfice, comme l'exige le quotidien clinique. Le modèle repose sur des critères internationalement reconnus, clairement définis et conformes à la jurisprudence suisse. Les présentations à des médecins et à l'industrie pharmaceutique ont reçu un accueil favorable. La distinction entre les évaluations d'études et les cas individuels ouvre de nouvelles perspectives pour l'avenir. und die bisherigen Modelle dem Vertrauensarzt einen grossen Handlungsspielraum ermöglichten.

\section{Die Zugangsgerechtigkeit im OLU-Bereich ist ungenügend}

Massnahmen wie die Revision von Art. 71a-d KVV durch das BAG, ein einheitliches Kostengutspracheformular [1] durch die Schweizerische Gesellschaft der Vertrauensund Versicherungsärzte (SGV) sowie die Verpflichtung der Versicherer, innert maximal 14 Tagen einen Antrag zu entscheiden, brachten hinsichtlich einheitlicher Beurteilung keine Fortschritte. Die SGV erkannte den Bedarf an einer stringenten, einheitlichen Nutzenbewertung und hat deshalb einer Arbeitsgruppe den Auftrag erteilt, ein neues Einheitsmodell zu entwerfen: OLUtool.

\section{OLUtool als Basis für die einheitliche Nutzenbeurteilung}

Die Arbeitsgruppe entwickelte unter Leitung der Vertrauensärzte Beat Seiler und Robert Fries das Einheitsmodell OLUtool in den zwei Varianten Onko und NonOnko. OLUtool-NonOnko wurde im Mai 2018 publiziert [2]. Nun folgt das Modell OLUtool-Onko. Beide Modelle zeichnen sich aus durch Transparenz und Praxisnähe mit klaren Regeln zur Anwendung. Dank den messbaren Kriterien liefert OLUtool bei verschiedenen Anwendern gleiche Ergebnisse. Das Modell berücksichtigt analog den ausländischen Bewertungsmodellen die 


\begin{tabular}{|c|c|}
\hline ASCO-Modell (USA) & $\begin{array}{l}\text { Nur solide Tumoren, nur Onkologie, nur RCT. Basis für Arzt-Patienten-Gespräch } \\
\text { zwecks Finanzierung der besten Therapie im Einzelfall }\end{array}$ \\
\hline ESMO-Modell (EU) & $\begin{array}{l}\text { Nur Onkologie und nur solide Tumoren. RCT und Studien mit geringer Evidenz. } \\
\text { Quantifizierung vorhanden }\end{array}$ \\
\hline EVITA (D) & Nur RCT, Algorithmus zur Anwendung ist kompliziert \\
\hline OLUtool $(\mathrm{CH})$ & $\begin{array}{l}\text { Ausgerichtet auf } \mathrm{CH} \rightarrow \text { Art. } 71 \mathrm{KVV} \text {. Alle Tumoren im Onko- und NonOnko-Bereich. } \\
\text { RCT und Arbeiten mit tiefer Evidenz sind berücksichtigt. Quantifizierbare und gut defi- } \\
\text { nierte Kriterien. Schnelle Beurteilung auch ohne Fachexperten und damit schnell und } \\
\text { dynamisch in der Beurteilung bei neuer Datenlage }\end{array}$ \\
\hline
\end{tabular}

patientenrelevanten Studienoutcomes sowie Surrogatmarker und Lebensqualitätsdaten. Zudem sind die Messkriterien und der Aufbau von OLUtool abgestimmt mit der neueren Rechtsprechung der Schweiz, welche sich zu Limiten von Überlebensdaten (OS) und Remissionen geäussert hat wie auch zu Beurteilungsschritten hinsichtlich Studien und Einzelfall. Das Modell trennt klar die Arbeitsschritte Studienrating und Einzelfall. Alle diese Faktoren sind wichtig, um die angestrebte Einheitlichkeit der Bewertung optimal zu erreichen. Der Erfolg einer raschen und kompetenten Bearbeitung hängt sehr stark von der Qualität des Gesuches durch die behandelnden Fachärzte ab. Von ihnen wird erwartet, dass sie begründen, warum kein Arzneimittel aus der Spezialitätenliste (SL) eingesetzt werden kann und wie der Patient zur Studienpopulation passt, sowie dass die Studie dem Begehren beigelegt ist. OLUtool-Onko basiert auf dem validierten Modell «Magnitude of Clinical Benefit Scale 1.1» [3] von ESMO (2017). Im Zentrum stehen HR und medianes Überleben (OS). Die Kriterien sind angepasst an den schweizerischen Art. 71a-d KVV. OLUtool-NonOnko basiert auf Erfahrungen der beiden Vormodelle MediScore und 9-Felder sowie Elementen aus dem ESMO-Modell 1.1. Im Zentrum stehen der Clinical Benefit sowie die Erreichung von Remissionen.

\section{SGV empfiehlt allen Vertrauensärzten das neue Modell zur Anwendung}

Es gibt die Meinung, dass nur ein Expertengremium eine korrekte Nutzenbeurteilung abgeben könne. Ein glaubwürdiges Expertengremium braucht transparente Beurteilungskriterien, was wiederum zu einem Modell führt. Zudem wird vergessen, dass Patienten und ihre Ärzte die Nutzenbeurteilung rasch bestimmt haben wollen, was ein Expertengremium für alle Fachgebiete der Medizin wohl kaum anbieten könnte. Im- merhin erfolgten im Jahr 2017 rund 30000 Begehren im Art. 71a-d KVV. Der Vorteil von OLUtool liegt gerade darin, dass kein Expertengremium zur Nutzenbestimmung nötig ist. Es kann von allen Vertrauensärzten angewendet werden, die sich in Interpretationen von Studien auskennen. Um in Zweifelsfällen dem Einzelfall möglichst gerecht zu werden, ist eine Rücksprache mit dem behandelnden Arzt empfehlenswert.

Zur Sicherstellung der korrekten Modellbeurteilung bietet die SGV ein Intensivseminar mit Zertifikat an.

\section{Neue Perspektiven dank OLUtool}

Aus der Dualität von OLUtool (Studie/Einzelfall) ergeben sich neue Perspektiven. Die Autoren regen an, ein nationales OLU-Center zu gründen. Im Center führen Vertrauensärzte mit Fachexperten die Studienratings durch, die als Basis der Einzelfallbeurteilung für die Vertrauensärzte der Versicherer zugänglich sind. Qualitätskontrolle und Validierung von OLUtool sind weitere Aufgaben des Centers. Die Gründung eines OLU-Centers ist ohne weitere Anpassung von behördlichen Regelungen möglich. Der Datenschutz wird nicht tangiert, da keine Patientendaten anfallen. Als Trägerschaft kommen das Bundesamt für Gesundheit (BAG), die SGV, Versicherungsverbände und "Grosskassen» oder im Onkobereich die Krebsliga in Betracht. Wünschbar ist, dass die Verwendung von OLUtool für die Nutzenbeurteilung durch das BAG als verbindlich erklärt wird.

Zusätzlich sind Leitlinien zu den Ausführungen im Art. 71 a-d KVV nötig. Diese fehlen heute, sind jedoch für die Zugangsgerechtigkeit unumgänglich. Grosse Interpretationen lassen folgende Begriffe zu: «Tödlicher Verlauf", "schwere und chronische gesundheitliche Beeinträchtigung» sowie «keine andere wirksame und zugelassene Behandlungsmethode».

Die Modelle sind abrufbar über die SGV-Homepage [4]. 